\title{
Manufacture and Adjustment of Radiation Dosimeter
}

\author{
Xiaochuan Sun, Hengxu Ma, Jinge Zhou \\ The College of Nuclear Technology and Automation Engineering, Chengdu University of Technology, Chengdu, China \\ Email: 1065761001@qq.com
}

How to cite this paper: Sun, X.C., Ma, H.X. and Zhou, J.G. (2020) Manufacture and Adjustment of Radiation Dosimeter. Open Access Library Journal, 7: e6574. https://doi.org/10.4236/oalib.1106574

Received: July 4, 2020

Accepted: October 23, 2020

Published: October 26, 2020

Copyright (c) 2020 by author(s) and Open Access Library Inc.

This work is licensed under the Creative Commons Attribution International License (CC BY 4.0).

http://creativecommons.org/licenses/by/4.0/

\begin{abstract}
The dose values of $\beta, \gamma$ and $x$ rays are important factors for evaluating the radioactivity in the environment, and monitoring the dose values of these rays in the environment is the primary measure to achieve radiation protection. Therefore, in view of the shortcomings of high power consumption and inconvenient carrying of some dosimeters at home and abroad, a new type of nuclear radiation dosimeter adapted to the new model was made, and the main parameters were measured. The dosimeter designed in this paper is a new type of nuclear radiation dosimeter mainly based on G-M counter tube and Atmega 8 main controller. This paper designs the circuit of the instrument and adjusts the parameters of the circuit to ensure the stability of the instrument and make the instrument work for a long time.
\end{abstract}

\section{Subject Areas}

Nuclear Technology and Application

\section{Keywords}

Radiation Protection, Nuclear Radiation Dosimeter, Monitor

\section{Introduction}

The radiation dosimeter produced this time has ultra-low power consumption, stronger processing power, faster processing speed, and richer on-chip resources. A Geiger-Miller counter is used to detect the intensity of beta particles, gamma rays, and X-rays. Compared with semiconductor detectors, it has high sensitivity, strong anti-interference ability, and high signal-to-noise ratio, so this nuclear radiation dosimeter can be used for users to provide smaller size, more stable work and longer continuous working time. In addition, the biggest difference between the radiation dosimeter and the ordinary dosimeter is that it can 
work continuously for a period of time, and store the measured radiation value at each time point, which can be easily viewed and operated later. In general, the characteristics of this nuclear radiation dosimeter are wide range, high accuracy, ultra-low power consumption, and the storage of radiation values in the time zone, which can provide a powerful guarantee for the monitoring of nuclear radiation [1].

\section{Overall Design Ideas}

\subsection{Instrument Design}

The radiation dosimeter uses the No. 5 battery to provide the working voltage for the entire system. The working voltage adopts the switch mode, which can realize the one-key turn-on voltage and one-key charging function. The design is novel and can convert the low voltage of the single-chip microcomputer to about $500 \mathrm{~V}$. The high voltage supplies power to the GM tube. The design of the power module highlights the characteristics of low power consumption and small size, which is an important module design [1].

This design instrument can have a real-time data display of the nuclear radiation situation, and give an alarm when the safety value is exceeded, and can call up the previously measured data for analysis at any time. This dosimeter has low power consumption and simple operation. It is suitable for work units with nuclear radiation and disaster-stricken areas. Because most nuclear radiation dosimeters have complex operations, high power consumption, poor continuous working energy, low accuracy, and are not suitable for nuclear radiation. Popularized in work units and disaster-stricken areas.

The instrument design is shown below (Figure 1):

\subsection{Calibration and Parameter Setting of the Dosimeter}

The calibration steps are as follows:

- Determine the source point on the field and mark it at different distances (within 4 meters) from this point.

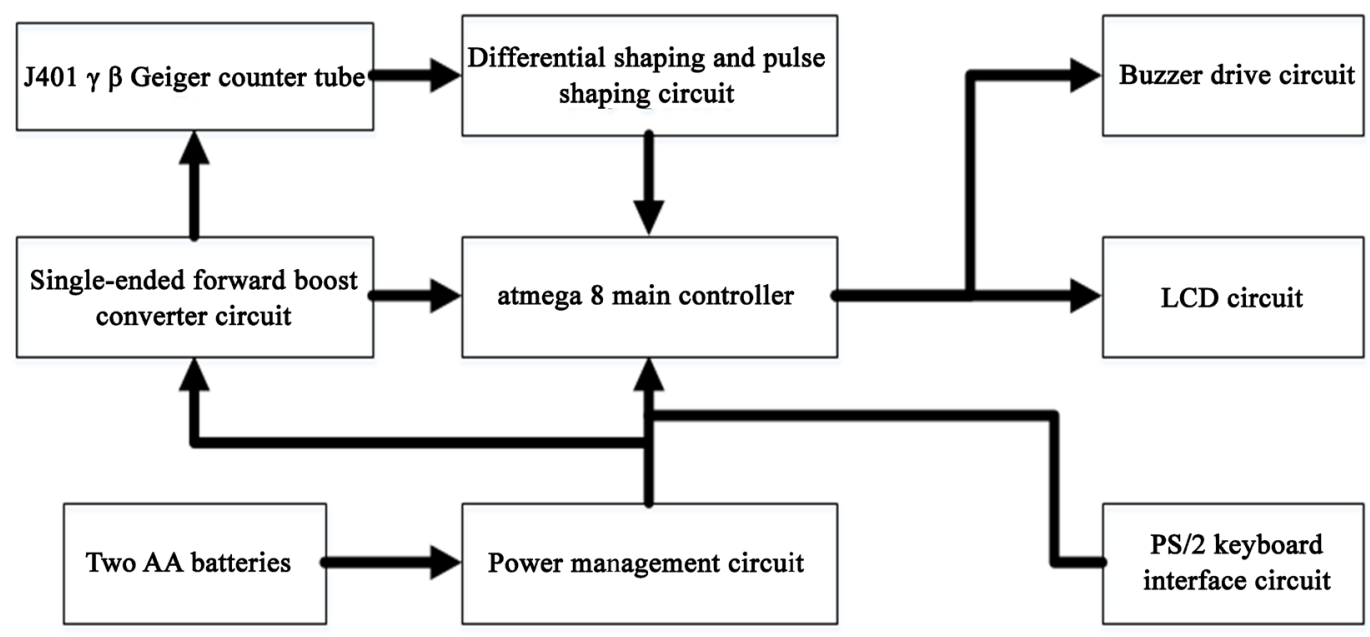

Figure 1. Schematic diagram of the overall design of the equipment. 
- Measure the background N0, set the measurement time to $100 \mathrm{~s}$, and take the average value of three measurements: $\mathrm{N} 01=33$, N04 $=34$, N07 $=34$. Place the radioactive source (No. 5 radium source) and measure the count rate $\mathrm{N}$ of each marked point separately.

- The dose rate at 1 meter from the source is: $\dot{D}_{1}=33.85 \dot{X}_{1}=60.783768 \times$ $10^{-8} \mathrm{~Gy} / \mathrm{h}$.

- Carry out curve fitting to get the calibration coefficient, and get the fitting curve: $y=a X+b=0.618 X-12.09$.

- Enter the calibration coefficients $a$ and $b(y=a X+b)$ into the corresponding dosimeter for testing.

\subsection{Parameter Input}

- Press the start button of the dosimeter and immediately press and hold the "Setting" button until the buzzer sounds and the system will enter the setting interface. If you do not enter the setting interface, restart the computer and continue to enter the above step dosimeter parameter input using sequential settings [2] [3].

- After entering the setting interface, the "Setting" key acts as a moving cursor, and the "Number" key acts as modifying the digits 0 - 9 at the cursor position.

- What needs to be set is the measurement time, which is generally set to $200 \mathrm{~s}$. Press the "Set" key to move the cursor and modify the numbers in sequence.

- After the time modification is completed, continue to move the cursor, the setting interface will enter, set the parameter a interface, enter the number of a.

- After the parameter b is set, continue to move the cursor, the setting interface will enter, set the parameter $b$ interface, you can enter the number of $b$, which may appear as a negative value of $b$, just set the + sign to-sign.

- Save all parameters. After saving, each dosimeter should be tested several times to compare the counts among all dosimeters without any abnormality.

\section{Design of Dosimeter Circuit}

\subsection{Power Module}

The circuit diagram is shown below (Figure 2):

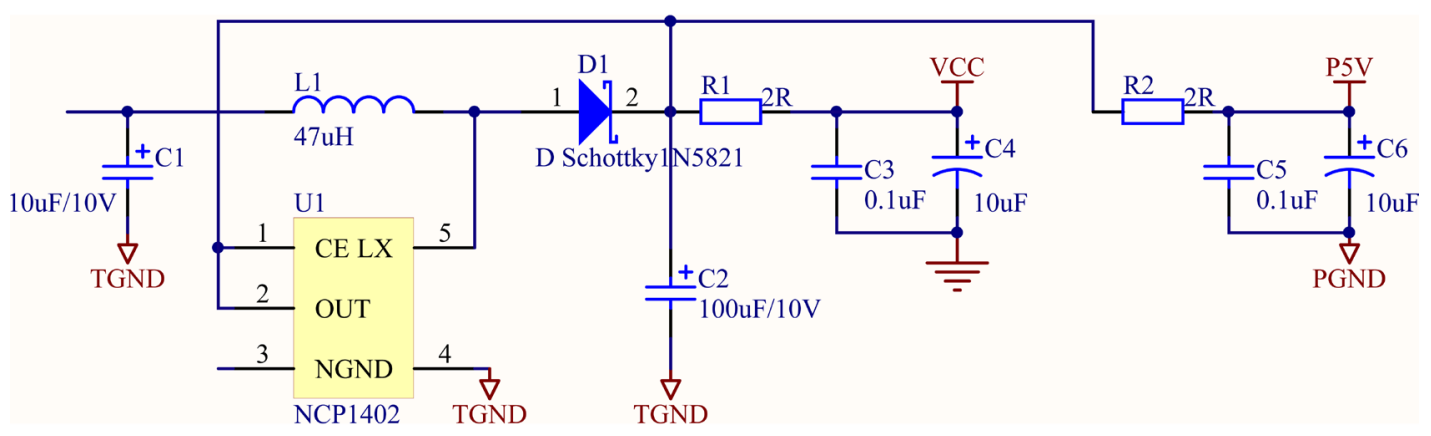

Figure 2. Power module schematic. 
When checking the power supply, from bottom to top, first measure the electrical parameters at the end of the power supply system, that is, the voltage at the left end of R2. From the measurement results, we found that the voltage on the left side of $\mathrm{R} 2$ is $5 \mathrm{~V}$, which meets the test requirements, that is, it is inferred that there is no problem with the power module.

\subsection{High Voltage Module}

The circuit diagram is shown below (Figure 3):

Use a universal meter to measure the voltage values on both sides of the transformer. Because the instrument uses JH401G-M Geiger counter tube, its recommended operating voltage is $400 \mathrm{~V}$, the plateau range is $360 \mathrm{~V}-440 \mathrm{~V}$, and the maximum operating voltage of the anode is $550 \mathrm{~V}$, so this article uses a single-ended forward converter based on PWM. By adjusting the duty cycle of the PWM wave to stabilize the output high voltage around $400 \mathrm{~V}$. The $400 \mathrm{~V}$ high voltage is sent to the At-mega 8 internal A DC for analog-to-digital conversion after being divided, and the duty ratio of the PWM wave is determined according to the conversion result.

\subsection{Manufacture and Adjustment}

- High voltage test: Before downloading the program to the hardware, we need to use the self-made high voltage debug board to debug the circuit board. The main purpose of this process is to check whether the low voltage $5 \mathrm{~V}$ and high voltage parts of the circuit board can work normally.

- Program debugging: first connect the circuit, press the power switch, turn on the high voltage meter, and check whether the high voltage is around $380 \mathrm{~V}$. If the high voltage display is not around $380 \mathrm{~V}$, you need to adjust the high voltage, re-modify the parameter download program, and finally modify the parameters.

- Welding of LCD screen: The dosimeter uses LCD802A liquid crystal display.

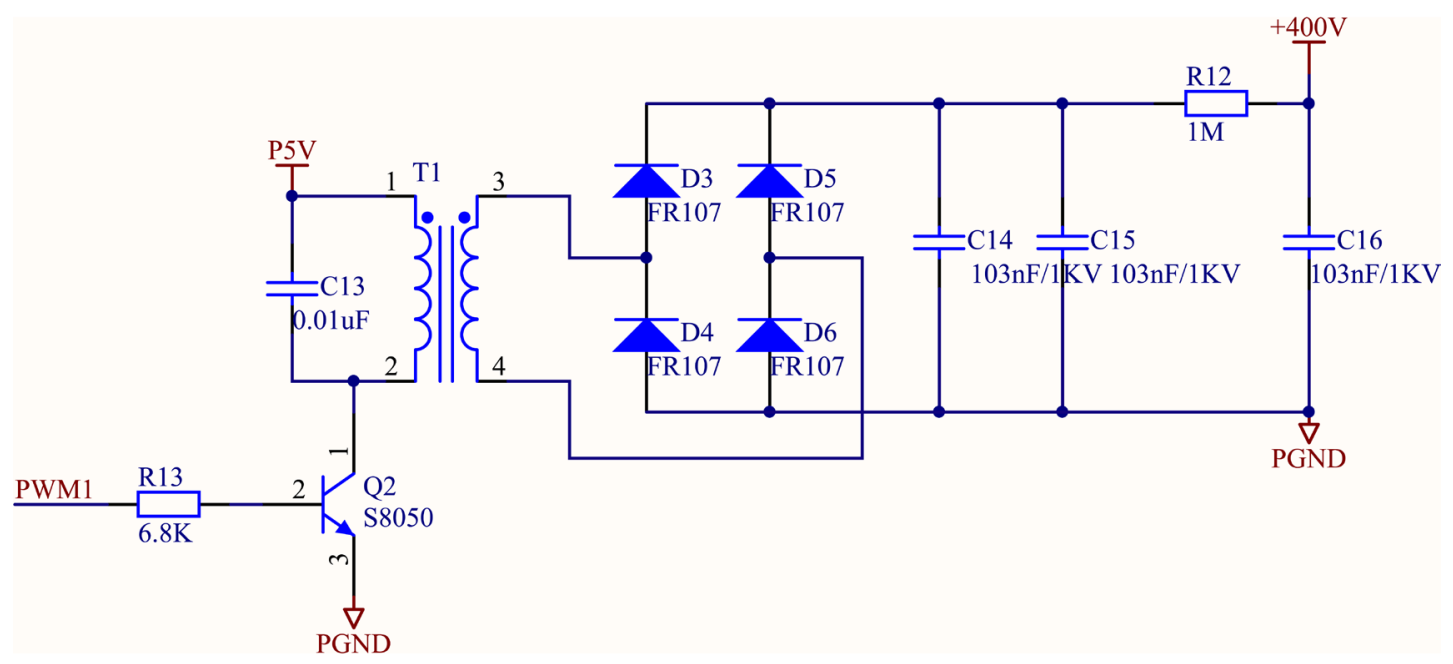

Figure 3. High voltage module schematic. 
To prevent short circuit before welding, the two capacitors on the back of the circuit board should be covered with black insulating tape; when welding the LCD panel, be sure to let the LCD panel The pad is close to the socket, and the LCD cannot be soldered too high.

\subsection{Machine Test}

- After the front and back covers of the dosimeter are installed, the battery can be installed and the whole machine is tested.

- Check if the power switch is turned on normally and turned off smoothly when turning on the power. If there is a button that cannot be pressed or does not spring, you need to re-install it; check whether the setting key S1 and number key S2 can be pressed normally Bounce, whether you can make settings and change numbers, if you cannot press or bounce, you need to re-install.

- Test the measurement stability of the dosimeter. The test time can be set to several dozen minutes. Check whether the count value is normal. If there is a sudden change or garbled number, you need to download the program again to adjust the high voltage.

- Possible problems and analysis of the whole machine test: the LCD screen has no display and the buzzer does not sound, it may be that the battery is not installed properly, or the battery is installed backwards. The measurement count result is 0 , it may be that the GM counter tube has a problem, and it needs to be re-checked after opening. If the check still shows no count, the GM counter tube has been damaged. During the measurement process, there is a sudden change in the measurement count, such as a sudden and rapid increase of hundreds or thousands, indicating that the high pressure of the GM counting tube is still not adjusted, and it needs to be readjusted and burned into the program [4].

\section{Conclusion}

The dosimeter designed this time is a new type of nuclear radiation dosimeter mainly based on G-M counter tube and Atmega 8 master controller. Compared with ordinary nuclear dosimeters, it has advanced features in portability, low power consumption and continuous operation. It can display a complete period of time in the amount of nuclear radiation. In this system, the Atmega 8 main controller is used to replace the traditional MCS-51 single-chip microcomputer. Its power consumption is extremely low, and its continuous working ability is stronger under the same power consumption.

\section{Acknowledgements}

My thesis work is not very mature, there are still many shortcomings. But this experience of doing a thesis has benefited me for life. I feel that doing a thesis is something that I really want to do with my heart. It is the process of my own 
learning and research. Without learning, there can be no research potential, and without my own research, there will be no breakthrough, then it is not called a thesis. I hope that this experience will enable me to continue to progress in my future studies. Here, thank everyone who helped me with my thesis.

\section{Conflicts of Interest}

The authors declare no conflicts of interest regarding the publication of this paper.

\section{References}

[1] Altunal, V., Guckan, V., Ozdemir, A. and Yegingil, Z. (2020) Radiation Dosimeter Utilizing Optically Stimulated Luminescence of BeO:Na, Tb, Gd Ceramics. Journal of Alloys and Compounds, 817, Article ID 152809.

https://doi.org/10.1016/j.jallcom.2019.152809

[2] Elin, V., Sharts, O. and Merkin, M. (2019) Mobile Radiation Dosimeter for Monitoring of Total Radiation On-Board of Civilian Aircraft. International Journal of Nuclear Safety and Security, 1, 64-84. https://doi.org/10.1504/IJNSS.2019.100129

[3] Ferraro, R., Danzeca, S., Brucoli, M., Masi, A., Brugger, M. and Dilillo, L. (2017) Design of a Radiation Tolerant System for Total Ionizing Dose Monitoring Using Floating Gate and RadFET Dosimeters. Journal of Instrumentation, 12, Aritle ID C04007. https://doi.org/10.1088/1748-0221/12/04/C04007

[4] Arar, D., Djeffal, F., Bentrcia, T. and Chahdi, M. (2014) New Junctionless RADFET Dosimeter Design for Low-Cost Radiation Monitoring Applications. Physica Status solid, 11, 65-68. https://doi.org/10.1002/pssc.201300146 